\title{
Rhodamine B pattern after whitening with peroxides and violet light: confocal laser microscopy observation
}

\section{Carolina Perches, Daylana P. Silva, Matheus Kury, Carolina B. André, Marcelo Giannini, Vanessa Cavalli}

\section{Abstract}

This study observed degradation pattern of Rhodamine B (RhodB) after whitening protocols with $35 \%$ hydrogen (HP) or $37 \%$ carbamide peroxide $(\mathrm{CP})$ combined with violet light $(\mathrm{VL})$. Bovine enamel-dentin blocks $(4 \times 4 \times 3 \mathrm{~mm})$ were immersed in $30 \mathrm{~mL}$ solution of $0.012 \mathrm{mM}$ Rhodamine B (Sigma-Aldrich, St Louis, MO, USA) during seven days at $80 \mathrm{C}$. Afterwards, specimens $(n=3)$ were submitted to whitening protocols: VL/HP, HP, VL/CP, CP and VL. Following application of gels/light on enamel surface, specimens were cut tinto 1-mm slices with vegetable oil. After polishing, slices were qualativetely evaluated by Laser Scanning Confocal Fluorescence Microscopy (TCS SP5AOBS, Leica Microsystems CMS GmbH, Germany) with a wavelength of $543 \mu \mathrm{m}$. Regardless of the peroxide presence, a thick layer of RhodB was preserved throughout enamel outer surface. Absence of fluorescence in the middle third of CP and HP groups indicates degradation of RhodB restricted to this area. Nevertheless, higher pattern of Rhodb degradation could be observed for groups combining CP or HP with VL. Even though VL presented a more homogenous pattern of RhodB distribution, the middle third seems to have been degraded by light irradiation. To conclude, RhodB penetrated through dental structures and it was preserved in the outer surface. However, peroxides were able to degrade it. Although light irradiation seems to have influenced RhodB patterns for CP and HP groups, violet irradiation alone did not completely degrade RhodB.

\section{Key words:}

Tooth Whitening, Peroxides, Light

\section{Introduction}

Application of violet light combined with high-concentrated peroxides has been investigated in literature since these protocols are being commonly reported by dentists. ${ }^{1}$

Such whitening procedures have raised concerns about intrapulpal concentration of hydrogen peroxide since light could accelerate degradation of free radicals. Even though these data has been recently reported, ${ }^{2}$ no confocal microscopy has been performed to analyse the pattern of RhodB degradation after whitening with violet LED.

Therefore, the aim of this study was to observe the degradation pattern of Rhodamine B (RhodB) after whitening protocols based on $35 \%$ hydrogen (HP) or $37 \%$ carbamide peroxide (CP) combined or not with violet light (VL).

\section{Results and Discussion}

Image 1. Summarized methodology employed in this study.

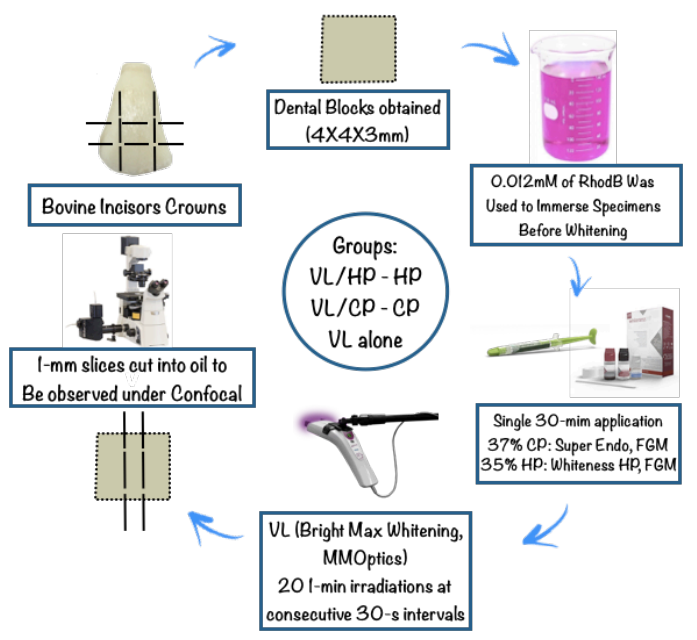

Image 2. Representative images obtained by confocal microscope.
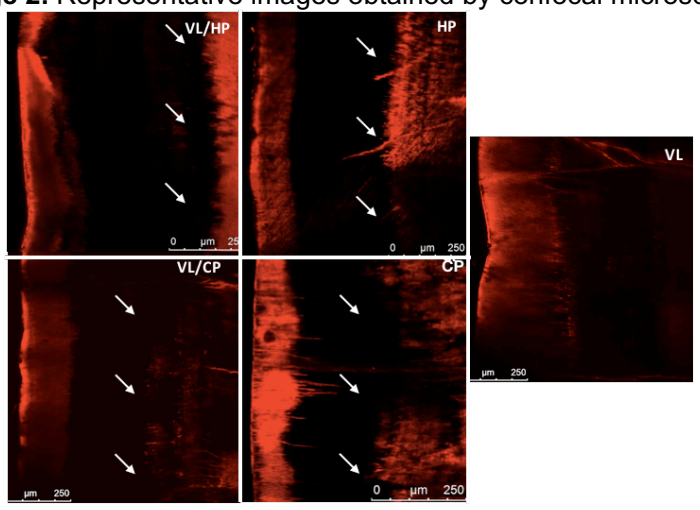

- White arrows in Image 2 indicates that degradation pattern for groups treated with peroxide and VL was depper than that oberserved for groups treated with $\mathrm{CP}$ and $\mathrm{HP}$.

- However, another research of our group ${ }^{2}$ showed that intrapulpal HP concentration of bovine teeth was not influenced by VL. In the same study, it was demonstrated that HP intra-chamber concentration was four times greater than CP's.

- Violet light irradiation may have influenced the observation of RhodB after whitening probably due to fact that visible light can interact with the solution ${ }^{3}$.

\section{Conclusions}

RhodB penetrated through dental structures and it was preserved in the outer surface. However, peroxides were able to degrade it. Although light irradiation seems to have influenced RhodB patterns for $\mathrm{CP}$ and HP groups, violet irradiation alone did not completely degrade RhodB.

Rastelli ANS et al. Violet LED with low concentration carbamide peroxide for dental bleaching: A case report. Photodiagnos Photodyn Ther. 2018 Sep; $23: 270-272$.
2 Perches C et al. Intrapulp peroxide concentration of teeth submitted to violet LED assisted in-office bleaching. [Congresso de

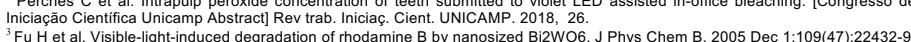

